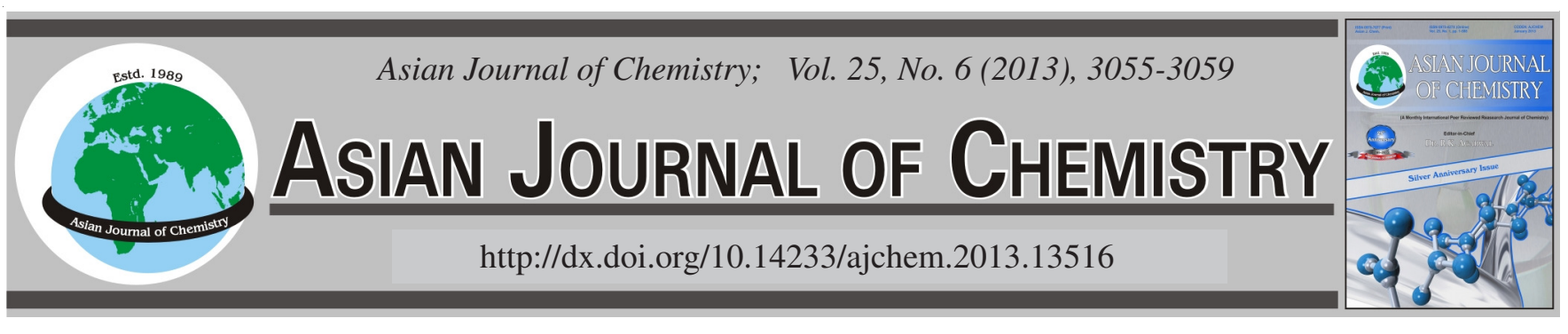

\title{
Densities and Refractive Indices for $N$-Arylhydroxamic Acids in Dimethylsulphoxide at Various Temperatures
}

\begin{abstract}
V. VERMA ${ }^{1, *}$ and R. PANDE ${ }^{2}$
${ }^{1}$ Agriculture Processing and Food Engineering, Faculty of Agricultural Engineering, Indira Gandhi Krishi Vishwavidyalaya, Raipur-492 006, India ${ }^{2}$ School of Studies in Chemistry, Pt. Ravishankar Shukla University, Raipur-492 010, India

*Corresponding author: E-mail: veenuverma88@gmail.com

(Received: 27 January 2012;

Accepted: 12 December 2012)

AJC-12517

Refractive index deviations $\left(\mathrm{n}^{\mathrm{E}}\right)$, excess volumes $\left(\mathrm{V}^{\mathrm{E}}\right)$ and molar refraction $\left(\mathrm{R}_{\mathrm{M}}\right)$ of two isomeric hydroxamic acids, $N$-phenyl-2methylbenzohydroxamic acid, $N$-phenyl-2-nitrobenzohydroxamic acid and $N$-phenyl-3-nitrobenzohydroxamic acid have been calculated from experimental data of refractive indices $(n)$ and densities $(\rho)$ in dimethyl sulphoxide at various temperatures $(298.15,303.15,308.15$ and 313.15) K. Results obtained have been discussed in terms of intermolecular ineteractions and a comprehensive discussion has been provided. The apparent molar volume at infinite dilution and the slope of Masson's equation are computed, to interpret the solute-solvent interaction.

Key Words: Density, Molar refraction, Hydroxamic acid.
\end{abstract}

\section{INTRODUCTION}

Density values are often reported together with refractive index for non-electrolyte as proof of purity of the samples ${ }^{1}$, The relation between refractive index and excess molar volumes has been studied for a long time, so it is common that these two properties are reported together.

$N$-aryl hydroxamic acids are $N$-acyl derivatives of hydroxamic acids represented by the general formula, $\mathrm{R}_{2}-\mathrm{CO}-$ $\mathrm{NR}_{1}-\mathrm{OH}$, where $\mathrm{R}_{1}$ and $\mathrm{R}_{2}$ are phenyl or substituted phenyl groups. It has been recognized as compounds of pharmacological, toxicological and pathological importance ${ }^{2-6}$.

Also the properties of dimethyl sulphoxide (DMSO) have been the subject of considerable interest because of its versatility as solvent and a plasticizer ${ }^{7}$. It is highly aprotic ${ }^{8}$ liquid which has the ability to participate in hydrogen bonding. The cryoprotectant effect of DMSO on biological and model membrane is widely recognized ${ }^{9-11}$. Thus it is main purpose of the work to study the relation between density and refractive index.

To this end, the densities and refractive indices of three hydroxamic acids i.e., $N$-phenyl-2-methylbenzohydroxamic acid (OMBHA), $N$-phenyl-2-nitrobenzohydroxamic acid (ONBHA) and $N$-phenyl-3-nitrobenzohydroxamic acid (MNBHA) have been measured under atmospheric pressure at various temperatures $(298.15,303.15,308.15$ and 313.15$)$ $K$. The experimental values of $\rho$ and $n$ were used to calculate the excess properties $\left(n^{\mathrm{E}}, \mathrm{R}_{\mathrm{M}}^{\mathrm{E}}, \mathrm{V}^{\mathrm{E}}\right)$, molar refraction $\left(\mathrm{R}_{\mathrm{M}}\right)$, polarizability $(\alpha)$ and apparent molar volume $\left(\mathrm{V}_{\phi}\right)$. These results have been used to understand the various types of interactions taking place in solutions of non-electrolyte and the temperature dependence of these interactions.

\section{EXPERIMENTAL}

All the three hydroxamic acids namely, $N$-phenyl-2methylbenzohydroxamic acid (I), $N$-phenyl-2-nitrobenzohydroxamic acid (II) and $N$-phenyl-3-nitrobenzohydroxamic acid (III), were prepared in this laboratory as reported in literature $^{12}$. These were purified by crystallization thrice with benzene and dried over phosphorus pentaoxide in vacuum desiccators for several hours. The melting point was determined on a tempo apparatus and is uncorrected. IR spectra were recorded with a FTIR 8400 Series Shimazdu (Japan) using $\mathrm{KBr}$ pellets. Elemental analysis was determined with a Vario-EL analysis apparatus. OMBHA (I), observed m.p. 81 ${ }^{\circ} \mathrm{C}$ and reported $81{ }^{\circ} \mathrm{C}$ in the literature, IR, $\mathrm{cm}^{-1}: 3050,1620$, 1340 and 915. Anal. $\left(\mathrm{C}_{14} \mathrm{H}_{13} \mathrm{NO}_{2}\right)$ calcd. C, 73.99; N, 6.16; H, 5.77. Found: C, 74.10; N, 5.95; H, 6.10. ONBHA (II), observed m.p. $148{ }^{\circ} \mathrm{C}$ and reported $148{ }^{\circ} \mathrm{C}$ in the literature, IR, $\mathrm{cm}^{-1}$ : 3100, 1612, 1349 and 950. Anal. $\left(\mathrm{C}_{13} \mathrm{H}_{10} \mathrm{~N}_{2} \mathrm{O}_{4}\right)$ calcd. C, 60.46; N, 10.85; H, 3.90. Found: C, $60.80 ; \mathrm{N}, 11.20 ; \mathrm{H}, 3.70$. and MNBHA (III), observed m.p. $117^{\circ} \mathrm{C}$ and reported $117^{\circ} \mathrm{C}$ in the literature, IR, $\mathrm{cm}^{-1}: 3100,1650,1340$ and 880. Anal. $\left(\mathrm{C}_{13} \mathrm{H}_{10} \mathrm{~N}_{2} \mathrm{O}_{4}\right)$ calcd. C, 60.46; N, 10.85; H, 3.90. Found: C, $60.65 ; \mathrm{N}, 10.80 ; \mathrm{H}, 4.00$. These were purified by crystallization 
thrice with benzene and dried over phosphorus pentaoxide in vacuum desiccators for several hours. DMSO of analytical grade was used for preparing hydroxamic acids solution of varying concentration from $(0.01$ to $0.1 \mathrm{M})$ by mass dilution technique. Uncertainty in solution concentration was estimated to be \pm 0.001 units.

Densities of hydroxamic acids in DMSO were determined by using a $10 \mathrm{~cm}^{3}$ double armed pycnometer at four temperatures $(298.15,303.15,308.15$ and 313.15$) \mathrm{K}$. The pycnometer was calibrated at desired temperature with freshly prepared triple distilled water. The estimate precision of density measurement of solution was $\pm 3 \times 10^{-5} \mathrm{~g} \mathrm{~cm}^{-3}$. The reproducibility of density measurement was $\pm 4 \times 10^{-5} \mathrm{~g} \mathrm{~cm}^{-3}$.

Refractive index was measured using thermostated Abbe's refractometer. The refractometer was calibrated by measuring the refractive indices of triply distilled water and toluene at known temperature ${ }^{13}$. The accuracy in the refractive index measurement was \pm 0.0001 unit. Temperature was controlled by circulating water around prisms of the refractometer from thermostatically controlled adequately stirred water bath (accuracy $\pm 0.1^{\circ} \mathrm{C}$ ). The sample mixtures were directly injected into the prism assembly of the instrument by means of an airtight hypodermic syringe. An average three to four measurements were taken for each sample mixture at various temperatures $(298.15,303.15,308.15$ and 313.15$) \mathrm{K}$. The experimental values of densities, $\rho_{0}$ and refractive index, $\eta_{0}$ of DMSO at 298.15, 303.15, 308.15 and $313.15 \mathrm{~K}$ are given in Table-1.

\begin{tabular}{|c|c|c|c|c|}
\hline \multicolumn{5}{|c|}{$\begin{array}{c}\text { TABLE-1 } \\
\text { PROPERTIES OF DMSO }\end{array}$} \\
\hline \multirow[b]{2}{*}{$\mathrm{T}(\mathrm{K})$} & \multicolumn{2}{|r|}{$\rho_{\mathrm{o}} / \mathrm{g} \mathrm{cm}^{-3}$} & \multicolumn{2}{|r|}{$\mathrm{n}_{\mathrm{o}}$} \\
\hline & $\begin{array}{l}\text { This } \\
\text { work }\end{array}$ & $\mathrm{L}$ & $\begin{array}{l}\text { This } \\
\text { work }\end{array}$ & $\mathrm{L}$ \\
\hline 298.15 & 1.0947 & $1.0955^{\mathrm{a}} ; 1.09475^{\mathrm{b}}$ & 1.4720 & $1.4771^{\mathrm{d}} ; 1.4765$ \\
\hline 303.15 & 1.0907 & $1.0896^{\mathrm{c}} ; 1.09076^{\mathrm{b}}$ & 1.4690 & $1.4752^{\mathrm{d}} ; 1.4740$ \\
\hline 308.15 & 1.0860 & $\begin{array}{l}1.0855^{\mathrm{a}} ; 1.08606^{\mathrm{b}} \\
1.0847^{\mathrm{c}}\end{array}$ & 1.4680 & $1.4720^{\mathrm{e}}$ \\
\hline 313.15 & 1.0804 & $1.08045^{\mathrm{b}} ; 1.0797^{\mathrm{c}}$ & 1.4660 & $1.4492^{\mathrm{d}} ; 1.4700$ \\
\hline
\end{tabular}

\section{RESULTS AND DISCUSSION}

Tables 2 and 3 list the densities and refractive indices of three hydroxamic acids in DMSO at four temperatures (298.15, 303.15, 308.15 and 313.15) $\mathrm{K}$.

From the density values, apparent molar volume, $\mathrm{V}_{\phi}$, is calculated using the following equation ${ }^{19}$,

$$
\mathrm{V}_{\phi}=1000\left(\rho_{0}-\rho\right) / c \rho_{0} \rho+\mathrm{M} / \rho
$$

where, $\rho$ and $\rho_{0}$ are the densities of solution and solvent, respectively. $\mathrm{M}$ is the molar mass of hydroxamic acids and $\mathrm{c}$ is the concentration of hydroxamic acids. The $V_{\phi}$ values are presented in Table-4. The positive values of $\mathrm{V}_{\phi}$ for all the hydroxamic acids indicate strong solute-solvent interactions. These interactions are strengthened with increasing concentration and temperature for $N$-phenyl-2-methylbenzo- (I), $N$ phenyl-3-nitro- (III) and weakened for $N$-phenyl-2-nitrobenzohydroxamic acid (II).

The apparent molar volume, $\mathrm{V}_{\phi}$, of hydroxamic acids is plotted against the concentration, $\mathrm{C}^{1 / 2}$, according to the Masson equation $^{20}$ :

$$
\mathrm{V}_{\phi}=\mathrm{V}_{\phi}^{0}+\mathrm{S}_{\mathrm{V}}^{*} \mathrm{C}^{1 / 2}
$$

where, intercept $\mathrm{V}^{0}{ }_{\phi}$ is the limiting apparent molar volume (same as partial molar volume at infinite dilution or standard partial molar volume), which is obtained by least-square fitting of $\mathrm{V}_{\phi}$ values using the above equation (2). $\mathrm{S}_{\mathrm{V}}{ }_{\mathrm{v}}$ is the experimental slope. The calculated $\mathrm{V}^{0}{ }_{\phi}$ and $\mathrm{S}_{\mathrm{V}}{ }_{\mathrm{v}}$ values along with their standard errors are listed in Table-5. The positive values $\mathrm{V}_{\mathrm{f}}^{0}$ for all the hydroxamic acids suggest strong solute-solvent interaction ${ }^{21}$. The $\mathrm{S}_{\mathrm{V}}{ }_{\mathrm{V}}$ values becomes large and positive for $\mathrm{N}$-phenyl-3-nitrobenzohydroxamic acid (III), suggest strong solute-solute interaction but opposite in case of N-phenyl-3methylbenzonydroxamic acid (I) and $N$-phenyl-2-nitrobenzohydroxamic acid (II) showing hydrophobic character ${ }^{22}$.

Form the measured values of refractive indices, molar refraction is calculated using the relation proposed by LorentzLorenz,

$$
\mathrm{R}_{\mathrm{M}}=\left[\left(\mathrm{n}^{2}-1 / \mathrm{n}^{2}+2\right)\right] \cdot \mathrm{V}
$$

In order to gain further information about specific interaction of any kind, the electronic polarizability of the system is computed. The molar refraction is related to the polarizabilty of molecules by Lorentz-Lorenz formula ${ }^{23}$,

\begin{tabular}{|c|c|c|c|c|c|c|c|c|c|c|}
\hline \multicolumn{11}{|c|}{$N$-phenyl-2-methylbenzohydroxamic acid } \\
\hline \multirow{2}{*}{ Temp. (K) } & \multicolumn{10}{|c|}{$\left.\mathrm{C}(\mathrm{mol} \mathrm{dm})^{-3}\right)$} \\
\hline & 0.01 & 0.02 & 0.03 & 0.04 & 0.05 & 0.06 & 0.07 & 0.08 & 0.09 & 0.10 \\
\hline 298.15 & 1.1073 & 1.1113 & 1.1165 & 1.1201 & 1.1254 & 1.1284 & 1.1314 & 1.1354 & 1.1394 & 1.1430 \\
\hline 303.15 & 1.1004 & 1.1033 & 1.1073 & 1.1133 & 1.1172 & 1.1189 & 1.1212 & 1.1272 & 1.1303 & 1.1356 \\
\hline 308.15 & 1.0951 & 1.0971 & 1.1000 & 1.1059 & 1.1078 & 1.1109 & 1.1129 & 1.1171 & 1.1204 & 1.1273 \\
\hline 313.15 & 1.0871 & 1.0898 & 1.0917 & 1.0986 & 1.1003 & 1.1021 & 1.1054 & 1.1091 & 1.1137 & 1.1193 \\
\hline \multicolumn{11}{|c|}{$\mathrm{N}$-phenyl-2-nitrobenzohydroxamic acid } \\
\hline 298.15 & 1.0958 & 1.1013 & 1.1081 & 1.1150 & 1.1176 & 1.1239 & 1.1285 & 1.1356 & 1.1416 & 1.1599 \\
\hline 303.15 & 1.0905 & 1.0958 & 1.1021 & 1.1099 & 1.1121 & 1.1172 & 1.1219 & 1.1287 & 1.1348 & 1.1553 \\
\hline 308.15 & 1.0876 & 1.0921 & 1.0994 & 1.1061 & 1.1091 & 1.1130 & 1.1181 & 1.1251 & 1.1321 & 1.1536 \\
\hline 313.15 & 1.0808 & 1.0860 & 1.0932 & 1.0997 & 1.1028 & 1.1069 & 1.1126 & 1.1188 & 1.1286 & 1.1464 \\
\hline \multicolumn{11}{|c|}{$\mathrm{N}$-phenyl-3-nitrobenzohydroxamic acid } \\
\hline 298.15 & 1.1579 & 1.1528 & 1.1469 & 1.1358 & 1.1201 & 1.1186 & 1.1124 & 1.1086 & 1.1020 & 1.0966 \\
\hline 303.15 & 1.1555 & 1.1505 & 1.1430 & 1.1321 & 1.1169 & 1.1136 & 1.1100 & 1.1036 & 1.0950 & 1.0907 \\
\hline 308.15 & 1.1519 & 1.1489 & 1.1411 & 1.1296 & 1.1140 & 1.1109 & 1.1065 & 1.1001 & 1.0903 & 1.0860 \\
\hline 313.15 & 1.1499 & 1.1466 & 1.1398 & 1.1279 & 1.1126 & 1.1090 & 1.1045 & 1.0982 & 1.0876 & 1.0817 \\
\hline
\end{tabular}

TABLE-2

DENSITY, $\rho\left(\mathrm{g} \mathrm{cm}^{-3}\right)$ OF THE HYDROXAMIC ACIDS AT VARIOUS TEMPERATURES 


\begin{tabular}{|c|c|c|c|c|c|c|c|c|c|c|}
\hline \multicolumn{11}{|c|}{$\begin{array}{c}\text { TABLE-3 } \\
\text { REFRACTIVE INDICES, n, OF HYDROXAMIC ACIDS AT VARIOUS TEMPERATURES }\end{array}$} \\
\hline \multicolumn{11}{|c|}{$N$-phenyl-2-methylbenzohydroxamic acid } \\
\hline \multirow{2}{*}{ Temp. (K) } & \multicolumn{10}{|c|}{$\mathrm{C}\left(\mathrm{mol} \mathrm{dm}^{-3}\right)$} \\
\hline & 0.01 & 0.02 & 0.03 & 0.04 & 0.05 & 0.06 & 0.07 & 0.08 & 0.09 & 0.10 \\
\hline 298.15 & 1.4720 & 1.4730 & 1.4745 & 1.4746 & 1.4749 & 1.4750 & 1.4754 & 1.4757 & 1.4757 & 1.4760 \\
\hline 303.15 & 1.4710 & 1.4715 & 1.4718 & 1.4720 & 1.4725 & 1.4725 & 1.4727 & 1.4735 & 1.4735 & 1.4745 \\
\hline 308.15 & 1.4689 & 1.4690 & 1.4691 & 1.4692 & 1.4692 & 1.4694 & 1.4695 & 1.4700 & 1.4700 & 1.4720 \\
\hline 313.15 & 1.4670 & 1.4673 & 1.4673 & 1.4676 & 1.4677 & 1.4677 & 1.4680 & 1.4685 & 1.4690 & 1.4700 \\
\hline \multicolumn{11}{|c|}{$\mathrm{N}$-phenyl-2-nitrobenzohydroxamic acid } \\
\hline 298.15 & 1.4743 & 1.4745 & 1.4750 & 1.4755 & 1.4760 & 1.4768 & 1.4770 & 1.4773 & 1.4775 & 1.4777 \\
\hline 303.15 & 1.4725 & 1.4730 & 1.4733 & 1.4740 & 1.4743 & 1.4748 & 1.4750 & 1.4755 & 1.4760 & 1.4765 \\
\hline 308.15 & 1.4714 & 1.4715 & 1.4720 & 1.4723 & 1.4725 & 1.4730 & 1.4735 & 1.4740 & 1.4750 & 1.4755 \\
\hline 313.15 & 1.4695 & 1.4700 & 1.4705 & 1.4705 & 1.4710 & 1.4715 & 1.4720 & 1.4725 & 1.4730 & 1.4735 \\
\hline \multicolumn{11}{|c|}{$N$-phenyl-3-nitrobenzohydroxamic acid } \\
\hline 298.15 & 1.4743 & 1.4745 & 1.4748 & 1.4749 & 1.4751 & 1.4754 & 1.4757 & 1.4760 & 1.4764 & 1.4769 \\
\hline 303.15 & 1.4725 & 1.4728 & 1.4731 & 1.4736 & 1.4739 & 1.4743 & 1.4747 & 1.4750 & 1.4753 & 1.4757 \\
\hline 308.15 & 1.4706 & 1.4710 & 1.4713 & 1.4717 & 1.4720 & 1.4725 & 1.4729 & 1.4734 & 1.4739 & 1.4742 \\
\hline 313.15 & 1.4690 & 1.4694 & 1.4697 & 1.4700 & 1.4706 & 1.4710 & 1.4713 & 1.4717 & 1.4720 & 1.4725 \\
\hline
\end{tabular}

TABLE-4

APPARENT MOLAR VOLUME, $\mathrm{V}_{\phi}^{0}\left(\mathrm{~cm}^{3} . \mathrm{mol}^{-1}\right)$ OF BOTH THE HYDROXAMIC ACIDS AT VARIOUS TEMPERATURES

\begin{tabular}{|c|c|c|c|c|c|c|c|c|c|c|}
\hline \multirow{3}{*}{ Temp. (K) } & \multicolumn{9}{|c|}{$N$-phenyl-2-methylbenzohydroxamic acid } & \\
\hline & \multicolumn{10}{|c|}{$\mathrm{C}\left(\mathrm{mol} \mathrm{dm}^{-3}\right)$} \\
\hline & 0.01 & 0.02 & 0.03 & 0.04 & 0.05 & 0.06 & 0.07 & 0.08 & 0.09 & 0.10 \\
\hline 298.15 & 193.24 & 188.49 & 182.38 & 178.11 & 171.92 & 168.37 & 164.82 & 160.10 & 155.39 & 151.22 \\
\hline 303.15 & 195.62 & 192.08 & 187.37 & 180.31 & 175.69 & 173.65 & 170.92 & 163.89 & 160.25 & 154.01 \\
\hline 308.15 & 197.70 & 195.36 & 191.84 & 184.82 & 182.65 & 178.96 & 176.63 & 171.66 & 167.73 & 159.53 \\
\hline 313.15 & 201.38 & 198.16 & 195.90 & 187.75 & 185.67 & 183.53 & 179.64 & 175.23 & 169.88 & 163.26 \\
\hline \multicolumn{11}{|c|}{$N$-phenyl-2-nitrobenzohydroxamic acid } \\
\hline 298.15 & 235.26 & 228.56 & 220.27 & 212.00 & 208.82 & 201.22 & 195.72 & 187.18 & 179.99 & 158.11 \\
\hline 303.15 & 235.71 & 229.32 & 221.67 & 212.22 & 209.58 & 203.40 & 197.73 & 189.51 & 182.21 & 157.73 \\
\hline 308.15 & 235.13 & 229.72 & 220.88 & 212.66 & 209.10 & 204.38 & 198.18 & 189.79 & 181.35 & 155.60 \\
\hline 313.15 & 237.69 & 231.35 & 222.61 & 214.65 & 210.89 & 205.95 & 199.06 & 191.55 & 179.75 & 158.39 \\
\hline \multicolumn{11}{|c|}{$N$-phenyl-3-nitrobenzohydroxamic acid } \\
\hline 298.15 & 160.52 & 166.53 & 173.63 & 186.88 & 205.78 & 207.64 & 215.12 & 219.73 & 227.67 & 234.22 \\
\hline 303.15 & 157.46 & 163.46 & 172.40 & 185.44 & 203.76 & 207.78 & 212.13 & 219.86 & 230.22 & 235.45 \\
\hline 313.15 & 154.20 & 158.18 & 166.28 & 180.61 & 199.02 & 203.44 & 208.81 & 216.44 & 229.37 & 236.54 \\
\hline
\end{tabular}

TABLE-5

PARTIAL MOLAR VOLUME, $\mathrm{V}_{\phi}^{0}$ AND SOLUTE-SOLUTE INTERACTION PARAMETER, $\mathrm{S}_{\mathrm{V}}^{*}$ OF THE HYDROXAMIC ACIDS AT VARIOUS TEMPERATURES

\begin{tabular}{|c|c|c|c|c|}
\hline $\mathrm{N}$-phenyl-2-methylbenzohydroxamic acid & \multicolumn{4}{|c|}{ ACIDS AT VARIOUS TEMPERATURES } \\
\hline & $298.15 \mathrm{~K}$ & $303.15 \mathrm{~K}$ & $308.15 \mathrm{~K}$ & $313.15 \mathrm{~K}$ \\
\hline $\mathrm{V}_{\phi}^{0}$ & 215.7253 & 218.3021 & 218.7563 & 222.3683 \\
\hline $\mathrm{S}_{\mathrm{V}}^{*}$ & -197.264 & -191.044 & -169.436 & -170.583 \\
\hline \multicolumn{5}{|c|}{$\mathrm{N}$-phenyl-2-nitrobenzohydroxamic acid } \\
\hline $\mathrm{V}_{\phi}^{0}$ & 274.1146 & 274.3388 & 274.7856 & 277.7446 \\
\hline $\mathrm{S}_{\mathrm{V}}^{*}$ & -317.79 & -313.473 & -316.484 & -322.929 \\
\hline \multicolumn{5}{|c|}{$\mathrm{N}$-phenyl-3-nitrobenzohydroxamic acid } \\
\hline $\mathrm{V}_{\phi}^{0}$ & 117.6816 & 112.0569 & 109.0075 & 104.0639 \\
\hline $\mathrm{S}_{\mathrm{V}}^{*}$ & 365.3631 & 386.0596 & 398.3157 & 406.0243 \\
\hline
\end{tabular}

$$
\mathrm{R}_{\mathrm{M}}=\frac{4 \pi \alpha \mathrm{N}}{3}
$$

where, $\alpha$ is the electronic polarizability and $\mathrm{N}$ is Avogadro's number. The value of $R_{M}$ and a are shown in Tables 6 and 7 for both hydroxamic acids. As inspection from Tables 6 and 7, the value of a for $N$-phenyl-3-nitrobenzohydroxamic acid (III) increases with concentration but apposite in case of $N$-phenyl-2-nitrobenzohydroxamic acid (II) and $N$-phenyl-2- methylbenzohydroxamic acid (I). This trend is slightly influence by temperature and the obvious decrease with temperature for $N$-phenyl-3-nitrobenzohydroxamic acids (III) but opposite in case of rest two hydroxamic acids, indicates the presence of intermolecular interaction between the molecules of solute and solvent.

Furthermore the refractive indices and densities experimental data, refractive index deviations defined on a mole fraction basis ${ }^{24} \mathrm{n}^{\mathrm{E}}$ and excess volume $\mathrm{V}^{\mathrm{E}}$, have been calculated as follows:

$$
\begin{gathered}
\mathrm{n}^{\mathrm{E}}=\mathrm{n}-\left(\mathrm{x}_{1} \mathrm{n}_{1}+\mathrm{x}_{2} \mathrm{n}_{2}\right) \\
\mathrm{V}^{\mathrm{E}}=\mathrm{V}-\left(\mathrm{x}_{1} \mathrm{~V}_{1}+\mathrm{x}_{2} \mathrm{~V}_{2}\right)
\end{gathered}
$$

where, $\mathrm{x}_{1}, \mathrm{n}_{1} \mathrm{~V}_{1}$ and $\mathrm{x}_{2}, \mathrm{n}_{2}, \mathrm{~V}_{2}$ is mole fraction, refractive index and molar volume of solvent and solute, respectively. $\mathrm{n}$ and $\mathrm{V}$ is the refractive index and molar volume of hydroxamic solutions. The values of $\mathrm{V}, \mathrm{n}^{\mathrm{E}}$ and $\mathrm{V}^{\mathrm{E}}$ are listed in Tables 8-10. According to Nakata and Sakurai the sign of $\mathrm{n}^{\mathrm{E}}$ is apposite to that of $\mathrm{V}^{\mathrm{E}}$, if the behaviour of refractive index is not linear between $\mathrm{n}_{1}$ and $\mathrm{n}_{2}$. This rule is truly fulfilled for compound (II) and (III) but not for (I) hydroxamic acids. The $N$-phenyl2-methylbenzohydroxmic acid (I) shows negative $\mathrm{n}^{\mathrm{E}}$ and $\mathrm{V}^{\mathrm{E}}$ values, indicates the heteromolecular interactions in the liquid 
mixtures and is attributed to charge transfer, dipole-dipole, dipole-induced dipole interactions and hydrogen bonding between the hydroxamic acids and DMSO. Compound (II) and (III) show positive $\mathrm{n}^{\mathrm{E}}$ and negative $\mathrm{V}^{\mathrm{E}}$ over the whole range of composition.
We can remark that the effect of the temperature is not significant, the $\mathrm{n}^{\mathrm{E}}$ value being bigger if the temperature increases for all the hydroxamic acids and $\mathrm{V}^{\mathrm{E}}$ values become smaller, when temperature increases.

TABLE-6

MOLAR REFRACTION, $\mathrm{R}_{\mathrm{M}}\left(\mathrm{cm}^{3} \cdot \mathrm{mol}^{-1}\right)$ OF HYDROXAMIC ACIDS AT VARIOUS TEMPERATURES

$\mathrm{N}$-phenyl-2-methylbenzohydroxamic acid

\begin{tabular}{|c|c|c|c|c|c|c|c|c|c|c|}
\hline \multicolumn{11}{|c|}{$N$-phenyl-2-methylbenzohydroxamic acid } \\
\hline \multirow{2}{*}{ Temp. (K) } & \multicolumn{10}{|c|}{$\mathrm{C}\left(\mathrm{mol} \mathrm{dm}^{-3}\right)$} \\
\hline & 0.01 & 0.02 & 0.03 & 0.04 & 0.05 & 0.06 & 0.07 & 0.08 & 0.09 & 0.10 \\
\hline 298.15 & 19.7839 & 19.7748 & 19.7628 & 19.7288 & 19.6730 & 19.6499 & 19.6373 & 19.6039 & 19.5600 & 19.5348 \\
\hline 303.15 & 19.8733 & 19.8645 & 19.8306 & 19.7577 & 19.7325 & 19.7282 & 19.7208 & 19.6702 & 19.6420 & 19.6106 \\
\hline 308.15 & 19.8926 & 19.8873 & 19.8640 & 19.7872 & 19.7806 & 19.7581 & 19.7524 & 19.7212 & 19.6875 & 19.6614 \\
\hline 313.15 & 19.9687 & 19.9575 & 19.9500 & 19.8630 & 19.8619 & 19.8563 & 19.8347 & 19.8125 & 19.7760 & 19.7385 \\
\hline \multicolumn{11}{|c|}{$\mathrm{N}$-phenyl-2-nitrobenzohydroxamic acid } \\
\hline 298.15 & 20.0825 & 20.0217 & 19.9481 & 19.8752 & 19.8780 & 19.8261 & 19.7840 & 19.7009 & 19.6343 & 19.3574 \\
\hline 303.15 & 20.1132 & 20.0678 & 19.9961 & 19.9125 & 19.9164 & 19.8745 & 19.8297 & 19.7580 & 19.7001 & 19.3944 \\
\hline 308.15 & 20.1265 & 20.0813 & 19.9988 & 19.9190 & 19.9056 & 19.8856 & 19.8435 & 19.7692 & 19.7122 & 19.3877 \\
\hline 313.15 & 20.1843 & 20.1393 & 20.0580 & 19.9707 & 19.9652 & 19.9417 & 19.8891 & 19.8275 & 19.7029 & 19.4409 \\
\hline \multicolumn{11}{|c|}{$\mathrm{N}$-phenyl-3-nitrobenzohydroxamic acid } \\
\hline 298.15 & 19.0036 & 19.1236 & 19.2637 & 19.4866 & 19.8009 & 19.8713 & 20.0266 & 20.1401 & 20.3098 & 20.4635 \\
\hline 303.15 & 18.9809 & 19.1043 & 19.2704 & 19.5051 & 19.8154 & 19.9220 & 20.0345 & 20.1958 & 20.4004 & 20.5311 \\
\hline 308.15 & 18.9739 & 19.0678 & 19.2390 & 19.4821 & 19.7997 & 19.9059 & 20.0323 & 20.2019 & 20.4379 & 20.5660 \\
\hline 313.15 & 18.9515 & 19.0505 & 19.2049 & 19.4508 & 19.7733 & 19.8861 & 20.0104 & 20.1744 & 20.4198 & 20.5859 \\
\hline
\end{tabular}

TABLE-7

POLARIZABILITY, $\alpha\left(\mathrm{cm}^{3} \mathrm{~mol}^{-1}\right)$ OF HYDROXAMIC ACIDS AT VARIOUS TEMPERATURES

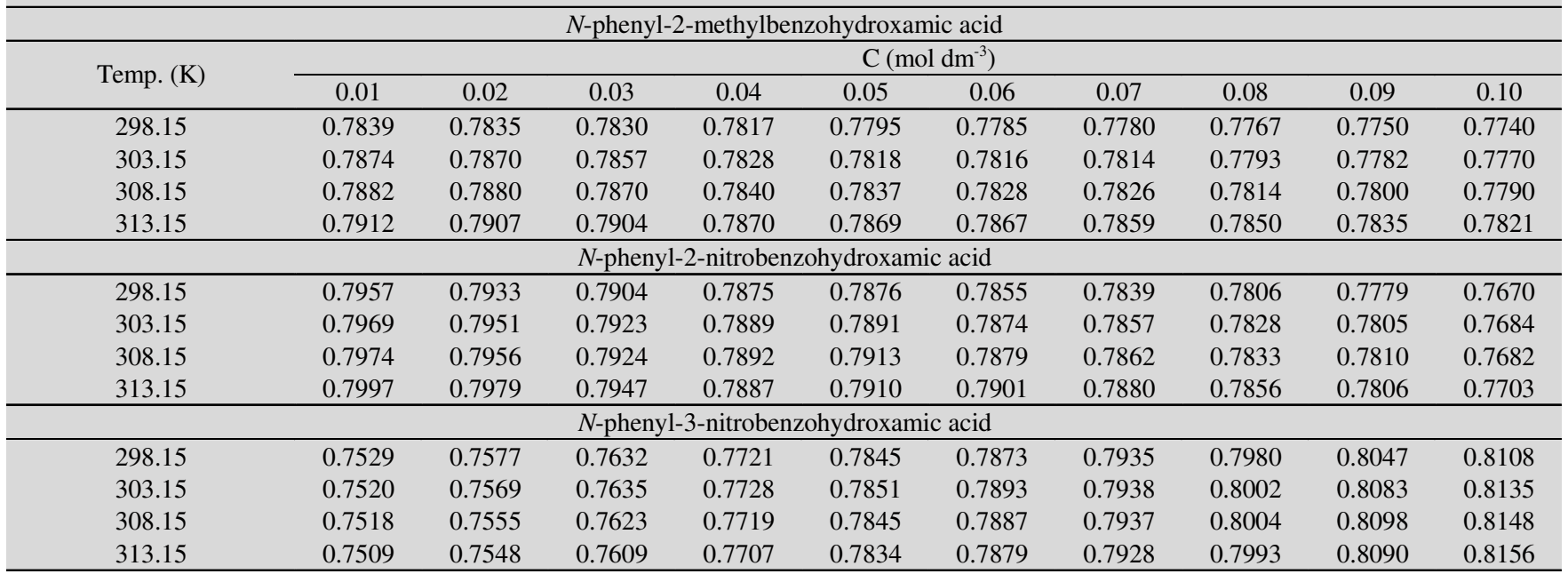

\section{TABLE-8}

MOLAR VOLUME, $\mathrm{V}\left(\mathrm{cm}^{3} \mathrm{~mol}^{-1}\right)$ OF HYDROXAMIC ACIDS AT VARIOUS TEMPERATURES

\begin{tabular}{|c|c|c|c|c|c|c|c|c|c|c|}
\hline \multirow{3}{*}{ Temp. (K) } & \multicolumn{9}{|c|}{$N$-phenyl-2-methylbenzohydroxamic acid } & \\
\hline & \multicolumn{10}{|c|}{$\mathrm{C}\left(\mathrm{mol} \mathrm{dm}^{-3}\right)$} \\
\hline & 0.01 & 0.02 & 0.03 & 0.04 & 0.05 & 0.06 & 0.07 & 0.08 & 0.09 & 0.10 \\
\hline 298.15 & 70.6518 & 70.4911 & 70.2577 & 70.1239 & 69.8880 & 69.7930 & 69.6983 & 69.5419 & 69.3862 & 69.2594 \\
\hline 303.15 & 71.1002 & 71.0039 & 70.8441 & 70.5580 & 70.4041 & 70.3888 & 70.3368 & 70.0548 & 69.9543 & 69.7164 \\
\hline 308.15 & 71.4427 & 71.4106 & 71.3137 & 71.0251 & 71.0013 & 70.8948 & 70.8613 & 70.6847 & 70.5640 & 70.2143 \\
\hline 313.15 & 71.9667 & 71.8865 & 71.8593 & 71.5067 & 71.4896 & 71.4692 & 71.3523 & 71.2072 & 71.0110 & 70.7467 \\
\hline \multicolumn{11}{|c|}{$N$-phenyl-2-nitrobenzohydroxamic acid } \\
\hline 298.15 & 71.4200 & 71.1779 & 70.8525 & 70.5300 & 70.4765 & 70.1915 & 70.0172 & 69.6855 & 69.4253 & 68.4215 \\
\hline 303.15 & 71.7624 & 71.5357 & 71.2414 & 70.8537 & 70.8292 & 70.6166 & 70.4319 & 70.1139 & 69.8455 & 68.7000 \\
\hline 308.15 & 71.9534 & 71.7788 & 71.4192 & 71.0954 & 71.0217 & 70.8863 & 70.6720 & 70.3436 & 70.0145 & 68.7998 \\
\hline 313.15 & 72.4109 & 72.1833 & 71.8262 & 71.5139 & 71.4288 & 71.2801 & 71.0273 & 70.7430 & 70.2348 & 69.2382 \\
\hline \multicolumn{11}{|c|}{$N$-phenyl-3-nitrobenzohydroxamic acid } \\
\hline 298.15 & 67.5829 & 67.9851 & 68.4460 & 69.2258 & 70.3167 & 70.5286 & 71.0415 & 71.4057 & 71.9554 & 72.4349 \\
\hline 303.15 & 67.7223 & 68.1256 & 68.6806 & 69.4541 & 70.5210 & 70.8490 & 71.1976 & 71.7322 & 72.4196 & 72.8313 \\
\hline 308.15 & 67.9320 & 68.2184 & 68.7931 & 69.6119 & 70.7080 & 71.0229 & 71.4221 & 71.9614 & 72.7365 & 73.1525 \\
\hline
\end{tabular}




\begin{tabular}{|c|c|c|c|c|c|c|c|c|c|c|}
\hline \multicolumn{11}{|c|}{$\begin{array}{r}\text { TABLE-9 } \\
\text { EXCESS MOLAR VOLUME } V^{\mathrm{E}}\left(\mathrm{cm}^{3} \mathrm{~mol}^{-1}\right) \text { OF HYDROXA }\end{array}$} \\
\hline \multicolumn{11}{|c|}{$N$-phenyl-2-methylbenzohydroxamic acid } \\
\hline \multirow{2}{*}{ Temp. (K) } & \multicolumn{10}{|c|}{$\mathrm{C}\left(\mathrm{mol} \mathrm{dm}^{-3}\right)$} \\
\hline & 0.01 & 0.02 & 0.03 & 0.04 & 0.05 & 0.06 & 0.07 & 0.08 & 0.09 & 0.10 \\
\hline 298.15 & -0.7621 & -1.0173 & -1.3443 & -1.5717 & -1.9002 & -2.0882 & -2.2757 & -2.5241 & -2.7713 & -2.9895 \\
\hline 303.15 & -0.7012 & -0.8935 & -1.1487 & -1.5291 & -1.7775 & -1.8879 & -2.0348 & -2.4089 & -2.6029 & -2.9322 \\
\hline 308.15 & -0.6305 & -0.7591 & -0.9518 & -1.3346 & -1.4532 & -1.6534 & -1.7805 & -2.0489 & -2.2612 & -2.6989 \\
\hline 313.15 & -0.4948 & -0.6736 & -0.7993 & -1.2486 & -1.3638 & -1.4821 & -1.6960 & -1.9374 & -2.2289 & -2.5871 \\
\hline \multicolumn{11}{|c|}{$N$-phenyl-2-nitrobenzohydroxamic acid } \\
\hline 298.15 & -0.0175 & -0.3772 & -0.8190 & -1.2568 & -1.4269 & -1.8259 & -2.1146 & -2.5580 & -2.9299 & -4.0322 \\
\hline 303.15 & -0.0624 & -0.4080 & -0.8200 & -1.3238 & -1.4662 & -1.7947 & -2.0949 & -2.5260 & -2.9072 & -4.1500 \\
\hline 308.15 & -0.1429 & -0.4372 & -0.9150 & -1.3560 & -1.5480 & -1.5348 & -2.1310 & -2.5731 & -3.0149 & -4.3263 \\
\hline 313.15 & -0.0736 & -0.4222 & -0.8990 & -1.3301 & -1.8007 & -1.8022 & -2.1719 & -2.5719 & -3.1915 & -4.2894 \\
\hline \multicolumn{11}{|c|}{$N$-phenyl-3-nitrobenzohydroxamic acid } \\
\hline 298.15 & -10.6441 & -10.3400 & -9.9786 & -9.3015 & -8.3178 & -8.2086 & -7.8020 & -7.5440 & -7.1038 & -6.7344 \\
\hline 303.15 & -4.0846 & -3.7844 & -3.3342 & -2.6685 & -1.7140 & -1.4950 & -1.2565 & -0.8353 & -0.2650 & 0.0317 \\
\hline 308.15 & -4.1462 & -3.9628 & -3.4932 & -2.7827 & -1.7995 & -1.5937 & -1.3055 & -0.8800 & -0.2236 & 0.0768 \\
\hline 313.15 & -4.4146 & -4.2123 & -3.8010 & -3.0658 & -2.1004 & -1.8579 & -1.5632 & -1.1431 & -0.4258 & -0.0168 \\
\hline
\end{tabular}

TABLE-10

EXCESS REFRACTIVE INDEX $\left(\mathrm{cm}^{3} \mathrm{~mol}^{-1}\right)$ OF HYDROXAMIC ACIDS AT VARIOUS TEMPERATURES

\begin{tabular}{|c|c|c|c|c|c|c|c|c|c|c|}
\hline \multicolumn{11}{|c|}{$N$-phenyl-2-methylbenzohydroxamic acid } \\
\hline \multirow{2}{*}{ Temp. (K) } & \multicolumn{10}{|c|}{$\mathrm{C}\left(\mathrm{mol} \mathrm{dm}^{-3}\right)$} \\
\hline & 0.01 & 0.02 & 0.03 & 0.04 & 0.05 & 0.06 & 0.07 & 0.08 & 0.09 & 0.10 \\
\hline 298.15 & -0.0020 & -0.0010 & 0.0005 & 0.0006 & 0.0009 & 0.0010 & 0.0014 & 0.0017 & 0.0017 & 0.0020 \\
\hline 303.15 & -0.0010 & -0.0005 & -0.0002 & 0.0000 & 0.0005 & 0.0005 & 0.0007 & 0.0015 & 0.0015 & 0.0025 \\
\hline 308.15 & -0.0011 & -0.0010 & -0.0009 & -0.0008 & -0.0008 & -0.0006 & -0.0005 & 0.0000 & 0.0000 & 0.0020 \\
\hline 313.15 & -0.0010 & -0.0007 & -0.0007 & -0.0004 & -0.0003 & -0.0003 & 0.0000 & 0.0005 & 0.0010 & 0.0020 \\
\hline \multicolumn{11}{|c|}{$N$-phenyl-2-nitrobenzohydroxamic acid } \\
\hline 298.15 & 0.0003 & 0.0005 & 0.0010 & 0.0015 & 0.0020 & 0.0028 & 0.0030 & 0.0033 & 0.0035 & 0.0037 \\
\hline 303.15 & 0.0005 & 0.0010 & 0.0013 & 0.0020 & 0.0023 & 0.0028 & 0.0030 & 0.0035 & 0.0040 & 0.0045 \\
\hline 308.15 & 0.0014 & 0.0015 & 0.0020 & 0.0023 & 0.0025 & 0.0030 & 0.0035 & 0.0040 & 0.0050 & 0.0055 \\
\hline 313.15 & 0.0015 & 0.0020 & 0.0025 & 0.0025 & 0.0030 & 0.0035 & 0.0040 & 0.0045 & 0.0050 & 0.0055 \\
\hline \multicolumn{11}{|c|}{$N$-phenyl-3-nitrobenzohydroxamic acid } \\
\hline 298.15 & 0.0003 & 0.0005 & 0.0008 & 0.0009 & 0.0011 & 0.0014 & 0.0017 & 0.0020 & 0.0024 & 0.0029 \\
\hline 303.15 & 0.0005 & 0.0008 & 0.0011 & 0.0016 & 0.0019 & 0.0023 & 0.0027 & 0.0030 & 0.0033 & 0.0037 \\
\hline 308.15 & 0.0006 & 0.0010 & 0.0013 & 0.0017 & 0.0020 & 0.0025 & 0.0029 & 0.0034 & 0.0039 & 0.0042 \\
\hline 313.15 & 0.0010 & 0.0014 & 0.0017 & 0.0020 & 0.0026 & 0.0030 & 0.0033 & 0.0037 & 0.0040 & 0.0045 \\
\hline
\end{tabular}

\section{Conclusion}

From density and refractive index studies of hydroxamic acids. It is revealed that: (a) Greater the value of $\mathrm{R}_{\mathrm{M}}$ and a for $\mathrm{N}$-phenyl-2-nitrobenzohydroxamic acid (II) and $\mathrm{N}$-phenyl-3nitrobenzohydroxamic acid (III), arises from polarization of phenyl ring when substitution group is $\mathrm{NO}_{2}$ rather than nonpolar $\mathrm{CH}_{3}$ group; (b) Negative $\mathrm{S}_{\mathrm{v}}^{*}$ values of $\mathrm{N}$-phenyl-2methylbenzohydroxamic acid (I) and $N$-phenyl-2-nitrobenzohydroxamic acid (II) shows hydrophobic character; (c) Positive $\mathrm{V}_{\phi}$, for all the hydroxamic acids suggest structure-maker with DMSO.

\footnotetext{
REFERENCES

1. S.C.P Hwa and W.T. Ziegler, J. Phys. Chem., 70, 2572 (1966).

2. H.K. Kehl, Chemistry and Biology of Hydroxamic Acids, Karger, Basel, (1982).

3. R.D. Wagh, H.S. Mahajan and S.G. Kaskhedikar, Asian J. Chem., 19, 4188 (2007).

4. S. Hanessian and S. Johnstone, J. Org. Chem., 64, 5896 (1999).

5. M. Whittaker, C.D. Floyd, P. Brown and A.J.H. Gearing, Chem. Rev., 99, 2735 (1999).

6. T. Kolasa, A.O. Stewart and C.D.W. Brooks, Tetrahedron:Asym., 7, 729 (1996).

7. F.A.J. Kerdesky, S.P. Schmidt, J.H. Holms, R.D. Dyer, G.W. Carter and D.W. Brooks, J. Med. Chem., 30, 1177 (1987).
}

8. R.K. Dewan, S.P. Gupta and S.K. Mehta, J. Soln. Chem., 18, 13 (1989).

9. A. Galmes, J. Besalduch, J. Bergay, A. Novo, M. Morey, J.M. Gureria and M.A. Duran, Transfusion, 39, 70 (1999).

10. T.T. Anchrodoguy. C.A. Cecchini and J.H. Crowe, Cryobiology, 28, 467 (1991).

11. T.J. Reid, G. Esteban, M. Clear and M. Gorogias, Transfusion, 39, 616 (1999).

12. R. Pande and S.G. Tandon, J. Chem. Eng. Data, 24, 72 (1979).

13. J.A. Riddick, W.B. Bunger and T. Sakano, Organic Solvent, Techniques of Chemistry, Wiley Interscience, New York, Vol I, edn. 4 (1986).

14. P.G. Sears, W.D. Sieqfried and D.E. Sunds, J. Chem. Eng. Data, 9, 261 (1964).

15. Bhanupriya, R.P. Rajwade and R. Pande, J. Eng. Chem. Data, 53, 1458 (2008).

16. M.A. Saleh, S. Akhtar, M.S. Ahmed and M.H. Uddin, Phys. Chem. Liq., 40, 621 (2002).

17. J.F. Casteel and P.G. Sears, J. Chem. Eng. Data, 19, 196 (1974).

18. S.A. Markarian and A.M. Erzyan, J. Chem. Eng. Data, 52, 1704 (2007).

19. I. Koltz and R.M. Rosenberg, Chemical Thermodynamics, Basic Theory and Methods, W.A. Benzanin: CA, edn 3 (1972).

20. D.O. Masson, Philos. Mag., 8, 218 (1929).

21. P. Brocos, A. Pineiro, R. Bravo and A. Amigo, Phys. Chem. Chem. Phys., 5, 550 (2003).

22. A. Ali, S. Sabir, A.K. Nain, S. Hyder, S. Ahmad and R. Patel, J. Indian Chem. Soc., 83, 581 (2006).

23. H. Lorentz, Ann. Phys., 9, 64; L.V. Lorenz, Ann. Phys., 11, 70 (1980).

24. Y.Y. Fialkov, Russ. J. Phys. Chem., 41, 398 (1967). 